\title{
Shock Acceleration in the Cluster of Galaxies ACO2163
}

\author{
Nceba Mhlahlo* \\ Wits University, South Africa \\ E-mail: nmgaxamba@gmail.com
}

L. Guennou

University of KwaZulu-Natal, King George V Ave, Durban, 4041, South Africa

E-mail: loic.guennou@gmail.com

\section{Feretti}

Istituto di Radioastronomia INAF, Via P. Gobetti 101, 40129 Bologna, Italy

E-mail: Iferetti@ira.inaf.it

The Mpc-scale diffuse radio emission that is observed in some of the merging galaxy clusters, both in the form of radio halos and relics, carries crucial information about the presence of nonthermal components in the intracluster medium, such as cosmic rays and magnetic fields, and about particle acceleration processes by shocks and turbulence. ACO2163, which is one of the clusters which show merging activity, is also one of the hottest and richest of the Abell galaxy clusters. The cluster has been observed countless times through many different wavelengths and has always shown remarkable properties.

In this paper we report on a discovery of a shock front in ACO2163, detected in the XMMNewton data, with a Mach number of $2.2 \pm 0.3$ obtained from temperature jump measurements. This Mach number is in good agreement with that implecated by the injection spectral index at the shock front from the radio observations, in combination with Diffusive Shock Acceleration.

We have also used the Kang \& Ryu model to explain the electrons at the shock front, and the observed discrepancy between the average spectral index of the halo and that predicted by the $M=$ 2.2 shock. In the scenario proposed by this model, the shock in ACO2163 is passing through a cloud of fossil relativistic electrons which were provided by a nearby radio galaxy, re-accelerating the fossil relativistic electrons in the fossil cloud, and will exit out of the cloud at cloud crossing time of $\sim 54$ Myr.

4th Annual Conference on High Energy Astrophysics in Southern Africa

25-27 August, 2016

Cape Town, South Africa

\footnotetext{
* Speaker.
} 


\section{The Merging Cluster ACO2163}

For fully virialised clusters formed through pure gravitational collapse, the mass and distribution of the hot gas trapped within their potential wells in the intracluster medium (ICM) are used to determine the X-ray luminosity, $L_{X}$, which is due to thermal bremsstrahlung emission, while the $\mathrm{X}$-ray temperature is determined by the depth of the potential well in which the ICM rests (1).

ACO2163 is a moderately distant $(\mathrm{z}=0.203(2))$, rich cluster, and is one of the hottest (mean $\mathrm{kT}=12$ $15.5 \mathrm{keV}$, (e.g. 3; 4; 5)) and most X-ray luminous $\left(L_{X}[2-10 \mathrm{keV}]=6 \times 10^{45} \mathrm{ergs}^{-1}\right.$, (6)). This cluster has been a subject of extensive studies at multiple wavelengths (see $3 ; 4 ; 7 ; 8 ; 9 ; 10 ; 11$, and references therein). Evidence for a recent merger that involves two or more components has been revealed by X-ray morphological studies of (3) based on ROSAT data, the spectroscopic analysis of (4) based on ASCA data, and that of (11) based on XMM-Newton and Chandra data.

Dynamical studies based on joint weak gravitational lensing and X-ray observations (7) have shown that ACO2163 is in a disturbed dynamical state, showing irregular distribution of mass and galaxies. This has been supported by weak-lensing studies of the dark matter distribution in ACO2163 which have suggested that a multiple merger is taking place in this cluster (12). Further evidence for a merger has come from X-ray temperature maps which have shown strong temperature variations and complex $\mathrm{X}$-ray thermal structure in their central regions $(8 ; 11)$. Temperature maps have also revealed a cold front in the south-west direction from the cluster centre associated with $\sim 8$ $\mathrm{keV}$ gas and surrounded by hotter $\sim 11 \mathrm{keV}$ gas (e.g. 13).

The merger scenario in ACO2163 is also supported by the presence of a radio halo in this cluster. The detection of a radio halo was first reported by (14). (9) re-observed the cluster at $1.4 \mathrm{GHz}$ with the Very Large Array (VLA), and the detection of one of the largest radio halos with a total extent of $\sim 11.5^{\prime}$ was reported (see also 15 , for low frequency observations). Diffuse emission at the N-E side of the halo (15) was also detected, and this feature was identified with a relic source.

Recent studies done by (11) which revealed striking similarities between ACO2163 and the 'Bullet' cluster have suggested the presence of a shock front. Evidence for a gas 'bullet' or cool-core separated from its galaxies and crossing the ACO2163 atmosphere along the east-west direction was presented. From evidence of pressure excess, particularly in the innermost regions of the main cluster ACO2163-A, (11) infered adiabatic compression of the gas in the ICM behind the westward moving gas 'bullet' as a result of presumed shock heating. This has suggested that the main-cluster in ACO2163 has accreted a subcluster along the east-west direction at a supersonic velocity. Though the merger event might have shocked the main-cluster atmosphere, however, there was no evidence for a shock front preceding this 'bullet', contrary to what is observed in the 'Bullet' cluster.

In this study we report a detection of a shock front in ACO2163 using the XMM-Newton data, and discuss possible acceleration mechanisms to explain the electrons at the shock front.

\section{X-ray Observations and the Shock Front}

The XMM-Newton observations of ACO2163 were done in August 2000. We retrieved the data of this cluster from the XMM-Newton Science Archive (obsID 0112230601, PI M. Turner, public data). The raw time of observation was $16.3 \mathrm{ks}$. The data were observed with the THIN1 
Figure 1: Bottom-left: Background-subtracted X-ray image of the cluster ACO2163 in the 0.5 $8 \mathrm{keV}$ energy range representing $\sim 9 \mathrm{ks}$ of data. The image has been smoothed with a Gaussian kernel with $F W H M=10^{\prime \prime}$. In the south-west direction we show the brightness edge that we argue is a shock, and the two lines near the bright emission region indicate the approximate extent of the edge. The linear scale bar has units counts/seconds. Bottom-right: Sectors demonstrating regions where spectra were extracted both toward the south-western direction and around the edge (top) and in the perpendicular direction (bottom).
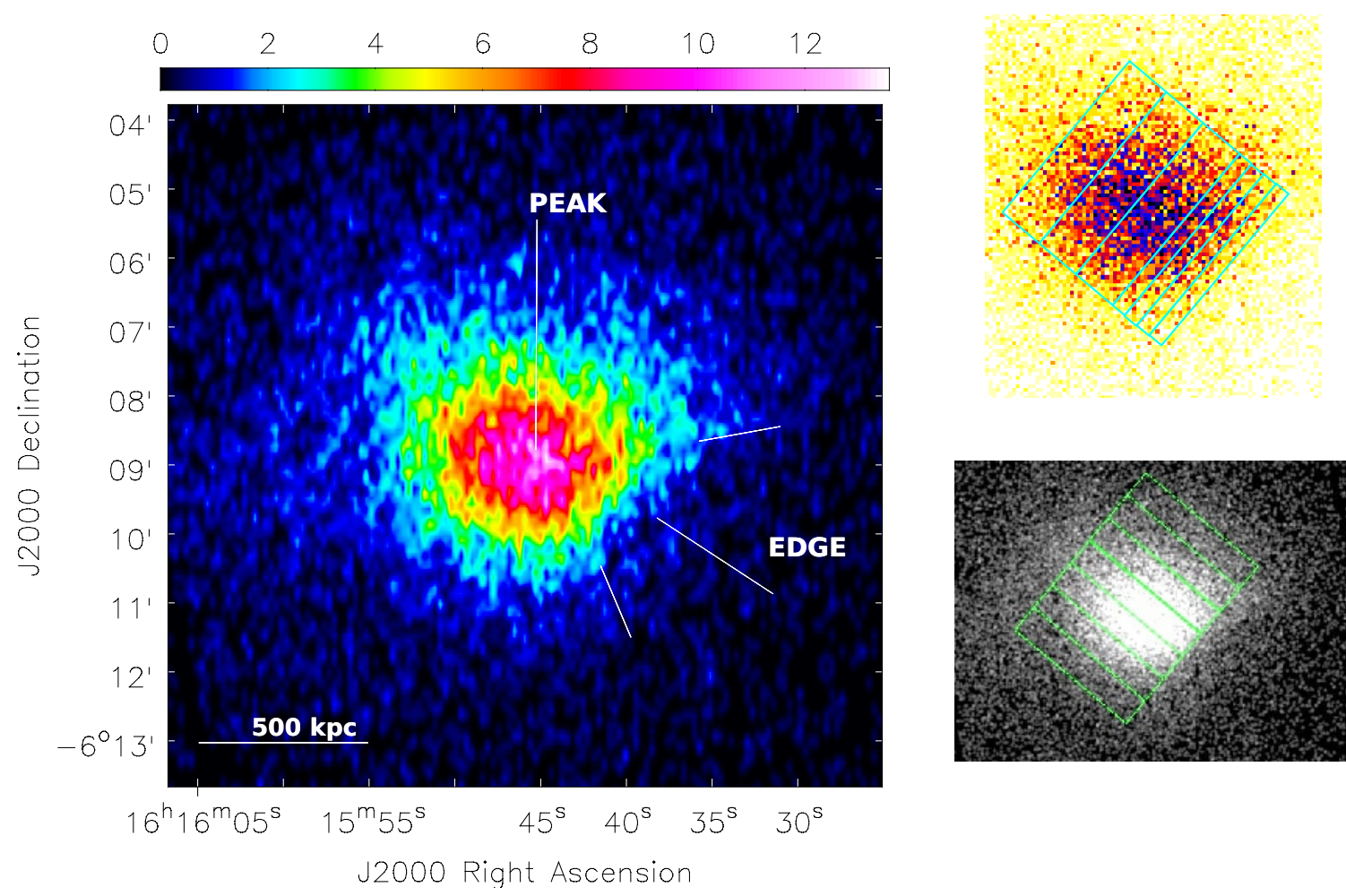

filter with the EPN and EMOS 1 and 2 cameras and then analysed using the SAS (Science Analysis System developed by the XMM-Newton team) tool from the Heasarc package to do the main part of the reduction. After removing the flares, we obtained a reduced image of $\sim 9 \mathrm{ks}$ for our study. We also obtained a blank field, representing the modelisation of the background to check the possible existence of hard/soft excess that could have altered the results obtained from our data, and no excess was observed.

In Figure 1 we show a smoothed image of the cluster, and we observe hints indicating the presence of a major merger, with the main axis of the cluster along the north-east, south west direction. The image clearly shows an edge to the south-west of the cluster with a clear cut in the otherwise elliptical form observed for this cluster. We have focused on this area, performing several checks on the existence of the shock.

We extracted several spectra to get an overview of the variation of temperature in the cluster. There is enough signal to divide the brightness region into seven bins (Fig. 1 - top-right panel). For three bins, the bin size was $163.34036^{\prime \prime} \times 39.6628^{\prime \prime}$. However, the bins around the edge (four bins) 

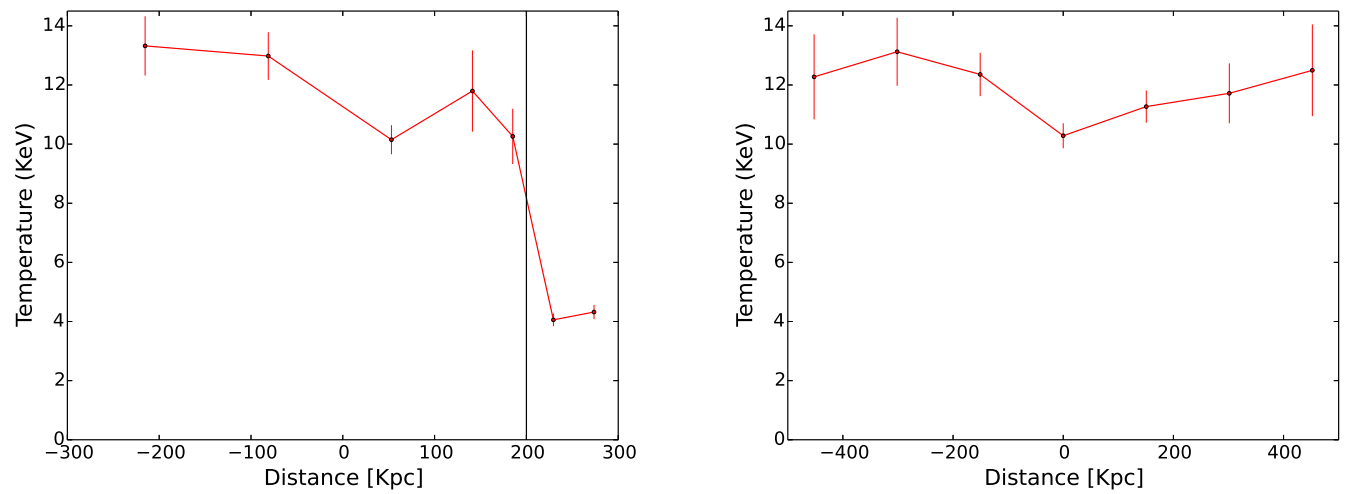

Figure 2: Plot of the temperature obtained along the main axis (left panel) and in the perpendicular direction (right panel). The vertical line represents the location where the shock is expected. The null distance represents the center of the cluster. The temperature obtained in the inner regions of the cluster is similar to the ones found in the literature (see e.g. 5; 11)

Table 1: Results from the fit of the temperature profile across the edge.

\begin{tabular}{cccc}
\hline Temperature $(\mathrm{keV})$ & Error $(\mathrm{keV})$ & Distance from centre $(\mathrm{kpc})$ & Reduced Chi-squared \\
\hline 13.322 & 1.002 & -215.336 & 0.999 for 762 degrees of freedom \\
12.979 & 0.807 & -81.183 & 1.031 for 976 degrees of freedom \\
10.149 & 0.492 & 52.993 & 0.936 for 1014 degrees of freedom \\
11.797 & 1.374 & 141.198 & 0.854 for 438 degrees of freedom \\
10.261 & 0.934 & 185.096 & 0.864 for 396 degrees of freedom \\
4.056 & 0.215 & 229.408 & 1.495 for 367 degrees of freedom \\
4.320 & 0.243 & 273.639 & 1.364 for 310 degrees of freedom \\
\hline
\end{tabular}

were made to be smaller (163.34036" x 13.2128"). This is ideal for minimizing contamination due to bright emission just inside the edge. We looked for the variations in temperature in the cluster using the combination of phabs and mekal models with the temperature, $\mathrm{H}$ density and abundance as free parameters, to fit the different spectra. We thus obtained a temperature profile along the main axis of the cluster to determine the gas temperature jump across the edge (see Fig. 2). The resulting temperature is shown in Table 1.

We also extracted, using the same method, spectra in the areas shown in the bottom-right panel of Fig. 1 to obtain the temperature variations behaviour in the northwest - southeast axis, perpendicular to the sector shown in the top-right panel of Fig. 1. The temperatures extracted from these sectors and the profiles are shown in Table 2 and in Fig 2 (right panel).

In the profile along the edge we see a sudden drop in temperature at the location of the brightness edge where we argue there is a shock (vertical line in Fig. 2), with a drop of more than $6 \mathrm{keV}$ between before and after the edge. However, in the case of the perpendicular profile, we observe temperatures which range between 10.283 and $13.124 \mathrm{keV}$ with no sudden drop in temperature in 
Table 2: Results from the fit of the temperature profile in the perpendicular direction.

\begin{tabular}{ccc}
\hline Temperature $(\mathrm{keV})$ & Error $(\mathrm{keV})$ & Reduced Chi-squared \\
\hline 12.274 & 1.438 & 0.998 for 379 degrees of freedom \\
13.124 & 1.151 & 0.945 for 641 degrees of freedom \\
12.356 & 0.733 & 0.846 for 970 degrees of freedom \\
10.283 & 0.422 & 0.998 for 1170 degrees of freedom \\
11.269 & 0.544 & 0.947 for 1081 degrees of freedom \\
11.718 & 1.013 & 0.848 for 679 degrees of freedom \\
12.495 & 1.553 & 0.740 for 364 degrees of freedom \\
\hline
\end{tabular}

this direction. This implies that the behaviour we observe in the area of the edge (sudden drop in temperature) is specific and is found only on the brightness edge of this galaxy cluster. Thus, the temperature profile confirms that the brightness edge is a shock front. The temperature jump of the right sign across the brightness edge leads to the ratio of the post-shock to pre-shock temperatures of 2.5 \pm 0.3 . After applying the Rankine-Hugoniot jump conditions across the shock (16), and assuming monoatomic gas with $\gamma=5 / 3$, we determine the Mach number:

$$
M=\left(\frac{(\gamma+1)^{2}\left(T_{2} / T_{1}-1\right)}{2 \gamma(\gamma-1)}\right)^{\frac{1}{2}}
$$

to be $2.2 \pm 0.3$.

\section{Radio Analysis}

We obtained the radio data from the ready-to-use maps of $(9 ; 15)$ that we used to read out spectral and flux profiles. These data were obtained at $1.4 \mathrm{GHz}$ and $0.3 \mathrm{GHz}$ with the VLA by $(9 ; 15)$ and show the detection of a giant radio halo, with a total extent of $\sim 11.5^{\prime}$ and an elongation in the E-W direction. The total fluxes in the halo were found to be $S_{1.4 \mathrm{GHz}}=155 \pm 2 \mathrm{mJy}$ and $S_{0.3 \mathrm{GHz}}=861 \pm 10 \mathrm{mJy}$, which led to an average spectral index $\alpha_{0.3}^{1.4}=1.18 \pm 0.04$.

A low resolution image of the radio halo at $1.4 \mathrm{GHz}$ is shown in Figure 3, and does not show any spacial coincidence between the shock and the radio halo edge. The shock is $\sim 290^{\prime \prime}(\sim 1000 \mathrm{kpc})$ from the edge towards the cluster centre, in the S-W direction. The approximate location of the shock is shown in the spectral index map of ACO2163 in Figure 4 (solid line).

As expected by electron re-acceleration models, regions influenced by the shock (and turbulence) should show spectral flattening, indicating the presence of more energetic radiating particles and/or a larger value of a local magnetic field strength (9). (15) identified two regions where they saw evidence of flat spectra in the spectral index map: the vertical region crossing the cluster centre (in the N-S direction) to a distance of $\sim 300^{\prime \prime}$ from the cluster centre, and the western halo region which was observed to be much flatter than the eastern region. The western region is where the shock is located. The flat spectra in the N-S region were interpreted as an indication of strong dynamical activity due to a merger occuring in the E-W direction. 


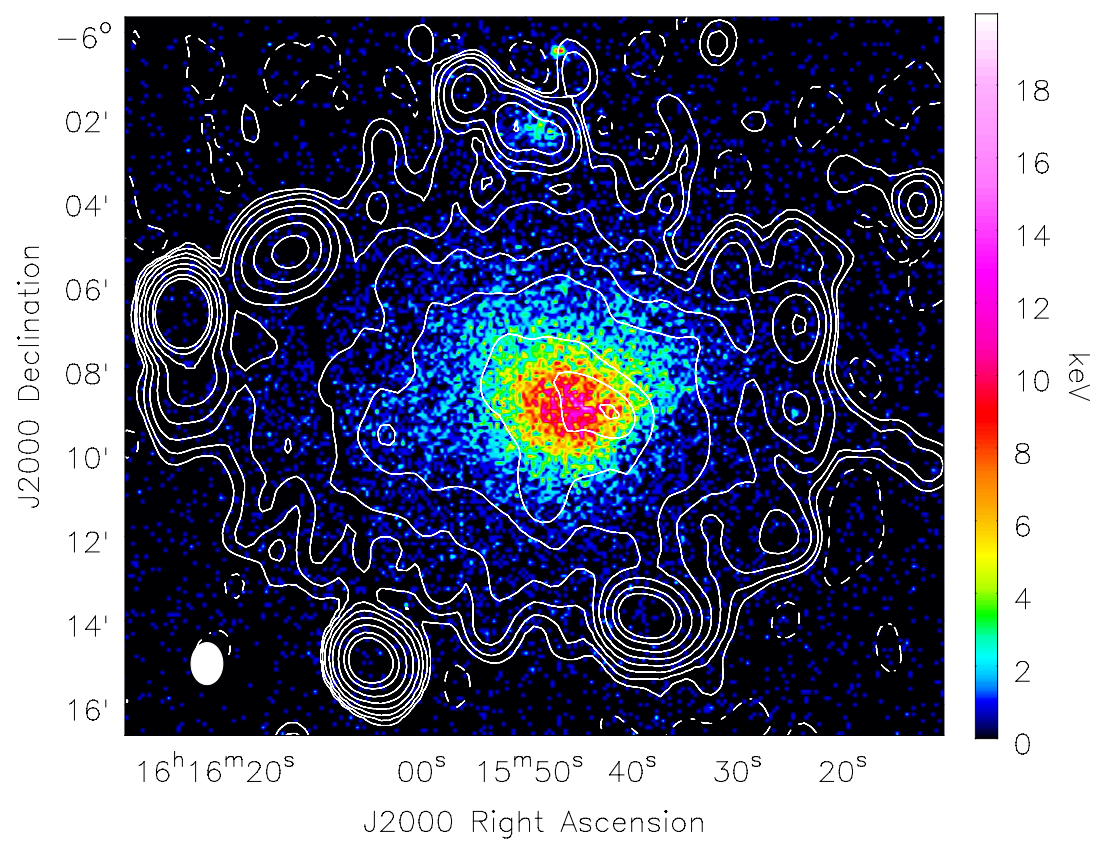

Figure 3: Radio contours of the halo in VLA at $1.4 \mathrm{GHz}$, with a resolution of $45^{\prime \prime} \times 60^{\prime \prime}$, are overlayed on the XMM-Newton X-ray image. The $\sigma$ noise level in the radio image plane is 0.03 $\mathrm{mJy} / \mathrm{beam}$. Contours are at $-3,3,6,12,24,48,96 \mathrm{mJy} / \mathrm{beam}$ times the noise level. The radio image is from (9).

We investigate if some of the flattening observed in the western direction could be due to the shock. In addition, we note the presence of a radio galaxy (J1615-061) behind the shock (Figure 4) at $\mathrm{RA}=16 \mathrm{~h} 15 \mathrm{~m} 41.3 \mathrm{~s}, \mathrm{DEC}=-06 \mathrm{~d} 09 \mathrm{~m} 08 \mathrm{~s}$, which was first reported by (9) (the source is labelled $\mathrm{T} 2$ in their Figure 2). If the radio galaxy is in the volume of the halo, mixing of the halo relativistic electrons with an aged electron population from the radio galaxy would change the shape of the halo spectrum, leading to a spectrum having a steep spectral index $(17 ; 18 ; 19)$.

To investigate if the flatter spectra in the S-W region could be due to the activity of the shock, and if there is any possible connection between the radio galaxy fossil electrons and the shock, we produced spectral index and flux radial profiles along the direction represented by the white dashed line in the spectral index image, emerging from the turbulent region near the cluster centre, passing through the radio galaxy location, to the halo edge.

Both the spectral index and flux profiles were obtained by averaging spectral index values from the spectral index image and flux values using the low resolution image, respectively, within small sectors along the direction marked by the dashed line. The sector area was made to be roughly equal to that of the beam. The spectral index profile (Figure 4-bottom-left panel) does not show any significant spectral variation but shows a globally flat spectral index.

As expected by electron re-acceleration models, regions influenced by the shock (and turbulence) or merger processes should show spectral flattening whereas spectral steepining is expected in presently undisturbed cluster regions (9). It is important to stress that though we do not detect any significant flattening at the location of the shock, there is no progressive steepining observed either 

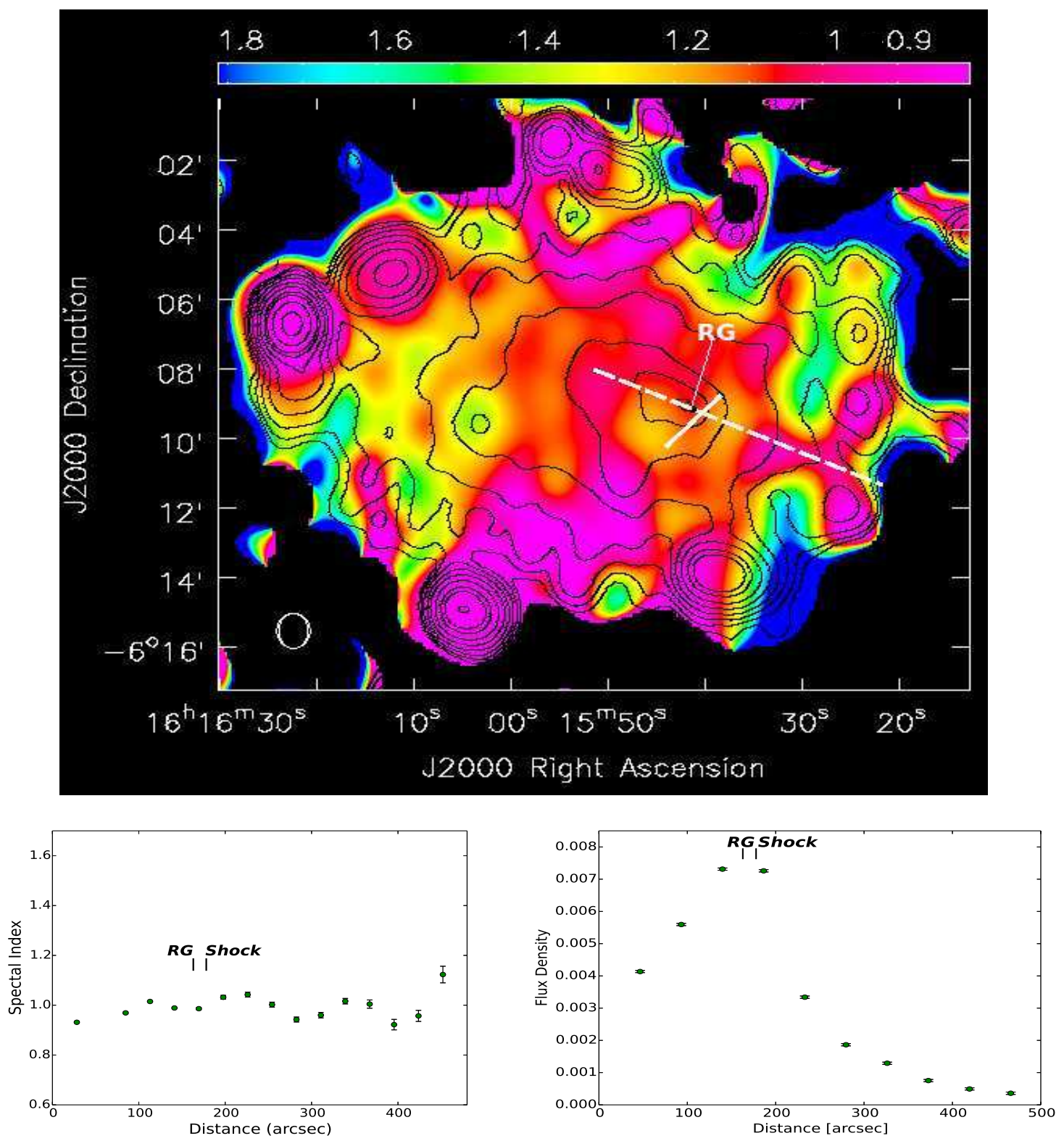

Figure 4: Top panel: Radio contours of the halo in VLA at $1.4 \mathrm{GHz}$, with a resolution of $45^{\prime \prime} \times 60^{\prime \prime}$, are overlayed on the colour-scale spectral index image between $0.3 \mathrm{GHz}$ and $1.4 \mathrm{GHz}$. The image is obtained with a resolution of $60^{\prime \prime} \times 51^{\prime \prime}$. The contours are at $-3,3,6,12,24,48,96 \mathrm{mJy} / \mathrm{beam}$ times the noise level. The location of the shock is represented by a solid white line and that of the radio galaxy is also indicated. The spectral index image is from (15). Bottom panels: Left: profile of the spectral index along the direction indicated by the dashed line segment that passes through the indicated location of the radio galaxy in the spectral index image. The origin of the distance scale starts near the approximate cluster centre at RA=16h15m51.2s, -06d08m01.6s. The locations of the radio galaxy and the shock are indicated. Right: The flux density distribution in the low resolution VLA radio image along the same dashed line segment shown in the spectral index image. 
on the western side of the cluster ACO2163. This could point to merging processes occuring on this side of the halo which produced the shock. The relatively globally flat spectral index could indicate the presence of more energetic radiating particles due to shock/merger activity in this region.

Another example of a Mach number $\sim 2$ shock which has not shown any evidence of spectral flattening at the shock location is observed in the cluster ACO665 (5; 15). Instead, progressive steepining with distance on the south-eastern side of the cluster where the shock is located, is observed and suggests that this cluster region is undisturbed by the merging processes and that shocks in major mergers are probably weak for particle acceleration (20).

The bottom-right panel of Figure 4 shows the flux density distribution along the dotted line segment passing through the radio galaxy region in the spectral index image. The flux density peaks at the location of the radio galaxy core behind the shock front, and rapidly declines on the side of the lobe (see 9), up to $\sim 250^{\prime \prime}$ from the core location.

\section{Discussion}

The main goal of this paper is to present evidence for the existence of a shock front in ACO2163, located at $\sim 175^{\prime \prime}(\sim 600 \mathrm{kpc})$ from the cluster center, on the south-western region of the halo (see Section 2). We have derived the Mach number associated with this shock from the direct measurement of a gas temperature jump. The temperature jumps from $4.1 \mathrm{keV}$ to $10.3 \mathrm{keV}$ by a factor of $2.5 \pm 0.3$ at the location of the shock front. This temperature jump corresponds to a Mach number $M=v_{s} / c_{s}$ with the value of $2.2 \pm 0.3$, where $c_{s}$ is a sound speed of $\sim 1021 \mathrm{~km} \mathrm{~s}^{-1}$. The shock velocity $v_{s}=M c_{s}$ has been determined to be $\sim 2238 \mathrm{~km} \mathrm{~s}^{-1}$.

A detection of a shock front in ACO2163 supports the results of (11) which revealed I) the 'bullet' nature of the supersonic infalling substructure in ACO2163, II) the westward motion of a stripped cool-core/'bullet' embedded in the hotter atmosphere of ACO2163-A, and III) adiabatic compression of the gas behind the 'bullet' due to shock heating. These observations point to the shock front being present in ACO2163, which we argue we have detected. The results of (11) also showed the similar nature of ACO2163 to that of the 'Bullet' cluster where a shock front associated with a two-component interaction was detected (21).

Given the location of the bullet which is at a projected distance of $290 \mathrm{kpc}$ from the main cluster (see 11), and assuming that the subcluster moves at the shock velocity, this velocity implies that the bullet crossed the main cluster nearly $0.1 \mathrm{Gyr}$ ago.

Our value of the Mach number is a typical value for a shock front in merging clusters ((see e.g. 22, for ACO520), (23, for ACO754), or (24, for CIZA2242, ACO3667, and ACO3376)), and since shock fronts are routinely observed in merging clusters, the detection of a shock in ACO2163 supports the results which show the merging nature of this cluster (see Section 1).

Radio observations of a number of merging galaxy clusters provide evidence for the presence of relativistic electrons and magnetic fields in the intracluster medium through the detection of synchrotron radio emission in the form of Mpc-scale sources called radio halos and relics (e.g. 25; 26). ACO2163 has one of the largest radio halos, indicating the presence of relativistic electrons in this cluster. Some of the relativistic electron population can be provided by Active Galactic Nuclei (AGNs) through radio jets or by previous episodes of shock/turbulence acceleration and be (re)accelerated by turbulence and shocks in the ICM. 
We have noted the presence of a radio galaxy along the path of the shock which might have provided the fossil radio plasma in the ICM for shock (re)-acceleration. This radio galaxy has a NRAO VLA Sky Survey (NVSS) flux of $16.57 \mathrm{mJy}$, but there is no spectral information about this source that is available. However, from our analysis there are two things we note about this source: (i) The radio galaxy core region fits the global trend of a flat spectrum, with a spectral index value of $\sim 1.0$ (Fig 4 ) and (ii) the flux density peaks at the location of the radio galaxy core. The relatively flat spectral index at the location of the radio galaxy and the high value of the flux could indicate either an ongoing radio galaxy activity and that the central activity of the core has not switched off, or that the radio galaxy fossil electrons were energized by the shock.

If the flux profile (Fig 4) traces the lobe from the radio galaxy core then the observed sharp decline in flux could point to a fading radio lobe. This could suggest that this is a dying radio galaxy and its previous cycle of activity might have provided the fossil radio plasma in the ICM which is reenergized by shock re-acceleration (27).

If one were to assume direct acceleration via Fermi mechanism of relativistic electrons by the M $\sim 2.2$ shock that we detect in ACO2163 (e.g. 28; 22), the spectral index in the shock region (accounting for down-stream losses due to inverse Compton and synchrotron energy losses) should be $\alpha=(p-1) / 2+0.5 \sim 1.53\left(\alpha=\alpha_{i n j}+0.5\right.$ and $\left.\alpha_{i n j}=(p-1) / 2 \sim 1\right)$, where $p$ is the slope of the energy spectrum of the electrons generated by the shock, and is related to the Mach number of the shock through

$$
p=2 \frac{M^{2}+1}{M^{2}-1}
$$

(e.g. 28). This spectral index value is not consistent with the total integrated spectral index $\alpha_{0.3}^{1.4}=$ $1.18 \pm 0.04$ obtained by (15), which predicts a high value of the Mach number ( 3.5). However, if we assume that the electrons from the radio galaxy are injected with $\alpha_{i n j} \sim 1$ (which is the average spectral index at the location of the shock - see Fig 4 - bottom-left panel), this injection spectral index in combination with Diffusive Shock Acceleration (DSA), would implicate a Mach number

$$
M_{\text {radio }}=\left(\frac{2 \alpha_{s}-3}{2 \alpha_{s}+1}\right)^{\frac{1}{2}}
$$

$\sim 2.2$, where $M_{\text {radio }}$ is the Mach number from the radio observations for a steady planar shock. This value of the Mach number is in excellent agreement with that derived from the X-ray observations, suggesting a steeper spectral index than that observed. This could mean that the electron population injected at the shock with $\mathrm{M} \sim 2.2$ results in a steeper spectrum with $\alpha \sim 1.6$ at higher frequencies.

The discrepancy between the observed integrated spectral index and that predicted by the shock (or between the corresponding Mach numbers) has been observed in another merging cluster 1RXS J0603.3+4214 (or the Toothbrush cluster - see $(19 ; 29)$ ). One of the possible explanations for the spectral index discrepancy has been provided by the model of (18) (see also (29)) which suggests shock re-acceleration in a cloud of a pre-existing population of cosmic-ray electrons. (18) have pointed out that the relation between the injection spectral index and the volume-integrated spectral index, $\alpha=\alpha_{i n j}+0.5$, should be applied only with caution in interpreting observed radio spectra. This is due to the observed increase in the integrated spectral index at high frequencies above $2.5 \mathrm{GHz}$, for some reported cases $(30 ; 31)$, which has been observed to be much stronger 
than expected for aging postshock electrons.

In the following discussion, as an alternative explanation to Fermi mechanism, and in attempting to address the observed discrepancy, we consider a scenario where the shock collides with a fossil electron cloud as detailed in $(17 ; 18)$ (hereafter the KR model or KR16), i.e. there could be a steep spectral curvature in the integrated spectrum above $\sim 2 \mathrm{GHz}$ which may not be interpreted by simple radiative cooling of postshock electrons.

In fact, (9) found a spectral index of $\alpha(i n t)_{1.365}^{1.465} \sim 1.6$ for the ACO2163 halo, between two nearby frequencies, and pointed out that even allowing for large errors, this could be an indication of a spectral steepening above $\sim 1.4 \mathrm{GHz}$.

According to the scenario proposed by KR16, if the shock has re-accelerated a pre-existing population of cosmic ray electrons provided e.g. by a radio galaxy, ACO2163 can possibly be explained by the spectral index ( $\alpha \sim 1.53$ ) which is predicted by the $M=2.2$ shock. Given that the shock is embedded in the halo, the above scenario (shock (re)-acceleration of AGN fossil electrons) could possibly lead to the production of an AGN relic. Since only 2-4\% polarization is detected (9) it is also likely that the relic (if any) at the X-ray shock is in the background and the cluster ICM is depolarizing.

In the models of KR for the Sausage relic, where a spherical shock sweeps through and then exits out of a finite-size cloud of fossil relativistic electrons, the spectrum of the re-accelerated fossil electrons follows a power-law with an exponential cutoff

$$
f_{\text {fossil }}(p)=f_{0} \times\left(\frac{p}{p_{\text {inj }}}\right)^{-s} \exp \left[-\left(\frac{\gamma_{e}}{\gamma_{e, c}}\right)^{2}\right]
$$

where $s=4.0-4.2$ is the slope, $\gamma_{e, c}=10^{3}-10^{4}$ is the cutoff Lorentz factor and $f_{0} \cdot p_{i n j}^{s}$ is a normalisation factor. We suggest that the main possible origin for such fossil electrons in the ICM would be the remnant of radio jet/lobe from the AGN, given that we observe a radio galaxy near the location of the shock. Considering that the spectral index of the radio galaxy lobe is also possibly about $\sim 1$ (Fig 4 ), which is steeper than that of typical radio galaxies, the power-law slope in Equation 4.3 would be $\sim 5$ in that case (Hyesung Kang, private communication). It should be mentioned that the parameters that (18) used in their model are relevant for the Sausage radio relic in cluster CIZA J2242.8+5301, and therefore the electron spectrum they predicted would be different from that of ACO2163, e.g., for their value of s, $\alpha_{i n j}=0.6$, which is flatter than the assumed $\alpha_{i n j}$ value in $\mathrm{ACO} 2163$.

If there is any interaction with the fossil electron cloud, it can be shown that the shock is approximately halfway within the lobe/cloud, currently re-accelerating fossil relativistic electrons, and will exit out of the cloud at cloud crossing time

$$
t_{\text {cross }} \sim 32.6 \mathrm{Myr}\left(\frac{L_{\text {cloud }}}{100 k p c}\right)\left(\frac{v_{s}}{3000 k m s^{-1}}\right)
$$

$\sim 54 \mathrm{Myr}$, which is the time it would have taken the shock to cross the entire cloud of fossil electrons. After crossing the fossil cloud, according to the KR model the re-acceleration of seed electrons is expected to stop. Thereafter the ensuing integrated radio spectrum steepens much more than predicted for aging postshock electrons. However, in ACO2163, due to previous merger activity, we suspect that the re-acceleration of electrons by the shock would not stop after $t_{\text {cross }}$. After 
the shock exits the cloud, further re-acceleration of fossil relativistic electrons, whose origin are electron populations that were (re)accelerated by turbulence during merger activities, would take place in the halo region. Thus considering that we do not observe the spectral steepening that is predicted by (18), the turbulent (re)acceleration is ongoing actively behind the shock (Fig 4).

Another alternative model for explaining the relativistic electrons at the shock front is the radio phoenix model of (32), where a fossil radio plasma is re-energised by a shock from a merger activity through adiabatic compression. Compression, by the shock, of fossil electrons in the ICM (see also 33; 23) is expected to significantly increase their synchrotron emission at the observing frequency and to produce radio emission in front of the bow shock. The first phase of the model of (32) which is used to explain radio relic emission involves injection by a radio galaxy.

Given the presence of a radio galaxy behind the shock, the scenario of adiabatic compression of fossil electrons at the shock front is also a likely possibility for ACO2163. In fact, (11) reported evidence of adiabatic compression of gas behind the crossing cool-core in ACO2163, possibly due to shock heating. In this scenario a radio phoenix is expected to result (32).

The adiabatic compression is expected to boost the lobe flux (32). The flux density profile passing through the radio galaxy region shows a sharp decline in flux, probably along the lobe, and that the flux density at the location of the radio galaxy core and the shock is $\sim 4$ times higher than that $80^{\prime \prime}$ ahead of the shock (Figure 4 - bottom right panel). It remains to be seen if this profile will change to indicate any boosting of flux along the lobe.

The possibility of a co-existence of a radio phoenix with an extended emission of the radio halo was revealed by the observations of ACO1033 (34), though the emission was faint. Bright emission and a steep and curved spectral index distribution are expected from such a source.

Our observations do not exclude other alternatives such as projection effects which could smooth out the temperature discontinuities, leading to a low value of the Mach number (29).

The mixing of the emission, most likely because of projection effects, can be an important factor that determines the spectral shape (19), and the emission at the location of the radio galaxy could be a mixture of the emission from the radio galaxy and the radio halo. This has an effect of changing the shape of the spectrum.

It should be stated that although the shock seems to be in the tail of the radio galaxy in $2 \mathrm{D}$ projected image, the radio galaxy and the X-ray shock may not overlap in 3D space. Since the radio galaxy looks undisturbed in (9), we should also consider the possibility that the X-ray shock is not interacting with the radio galaxy in $\mathrm{ACO} 2163$, i.e. there are projection effects. But since the radio halo should be approximately spherical and is very extended, the shock should occur within the radio emitting region.

ACO2163 joins other shocks with similar or lower Mach numbers which do not show a relic, such as A521 $(M=2.5 ;(35 ; 36))$, A754 $(M=1.6 ;(37 ; 23))$, A3667 $(M=2 ;(38))$, A665 (M 3; (39)), A2034 ( $M \sim 1.6$; $(40)$, and the eastern shock in the Bullet cluster $(M=2.5 ;(41))$.

\section{Summary and Conclusion}

In this paper we have presented a combined X-ray and radio study of the nearby galaxy cluster ACO2163.

These are the main findings from our study and analysis: 
1. The XMM-Newton observations have confirmed the presence of a shock front south-west of the cluster centre in $\mathrm{ACO} 2163$. We have estimated the Mach number from the X-ray temperature ratio before and after the shock, and have found a value of $M=2.2 \pm 0.3$. This is a typical value for a shock front in a galaxy cluster, and supports the results showing the merging nature of ACO2163.

2. We observe a globally flat spectral index on the south-western side of the cluster where the shock is located, suggesting the presence of energetic electrons in this region.

3. We find a discrepancy between the observed average spectral index and that predicted by the shock (or between the corresponding Mach numbers). The average spectral index in the region of the shock predicts a Mach number $M=3.5$ which is higher than that observed in most merging clusters.

4. In combination with DSA, a steep spectral index $(\alpha \sim 1.6)$ which was observed for two nearby frequencies in $\mathrm{ACO} 2163$, implecates a Mach number $M=2.2$, in agreement with our X-ray results.

5. We have attempted to explain this discrepancy through the KR model which predicts a steep spectrum of the re-accelerated fossil electrons at high frequencies for ACO2163, much steeper than that of the Sausage relic.

6. Following the KR model, we show that if the shock is interacting with the radio galaxy cloud of fossil electrons, then the shock is currently in the tail of the radio galaxy, re-accelerating the fossil relativistic electrons, and will exit the cloud after cloud crossing time of $\leq 54 \mathrm{Myr}$.

7. Alternative explanations include mixing of the emission, most likely because of projection effects, which can be an important factor that determines the spectral shape.

\section{References}

[1] G. W. Pratt, J. H. Croston, M. Arnaud and H. Böhringer, Galaxy cluster X-ray luminosity scaling relations from a representative local sample (REXCESS), Astronomy and Astrophysics 498 (May, 2009) 361-378, [0809.3784].

[2] M. F. Struble and H. J. Rood, A Compilation of Redshifts and Velocity Dispersions for ACO Clusters, Astrophysical Journal Supplement Series 125 (Nov., 1999) 35-71.

[3] D. Elbaz, M. Arnaud and H. Boehringer, The gas distribution and binding mass in the A 2163 cluster., Astronomy and Astrophysics 293 (Jan., 1995) .

[4] M. Markevitch, R. Mushotzky, H. Inoue, K. Yamashita, A. Furuzawa and Y. Tawara, Abell 2163: Temperature, Mass, and Hydrostatic Equilibrium, Astrophysical Journal 456 (Jan., 1996) 437, [astro-ph/9506090].

[5] M. Markevitch and A. Vikhlinin, Merger Shocks in Galaxy Clusters A665 and A2163 and Their Relation to Radio Halos, Astrophysical Journal 563 (Dec., 2001) 95-102, [astro-ph/0105093].

[6] M. Arnaud, J. P. Hughes, W. Forman, C. Jones, M. Lachieze-Rey, K. Yamashita et al., A2163 - an exceptionally hot cluster of galaxies, Astrophysical Journal 390 (May, 1992) 345-349. 
[7] G. Squires, D. M. Neumann, N. Kaiser, M. Arnaud, A. Babul, H. Böhringer et al., Weak Gravitational Lensing and X-Ray Analysis of Abell 2163, Astrophysical Journal 482 (June, 1997) 648-658, [astro-ph/9603050].

[8] F. Govoni, M. Markevitch, A. Vikhlinin, L. van Speybroeck, L. Feretti and G. Giovannini, Chandra Temperature Maps for Galaxy Clusters with Radio Halos, Astrophysical Journal 605 (Apr., 2004) 695-708, [astro-ph / 0401421 ].

[9] L. Feretti, R. Fusco-Femiano, G. Giovannini and F. Govoni, The giant radio halo in Abell 2163, Astronomy and Astrophysics 373 (July, 2001) 106-112, [astro-ph/ 0104 451].

[10] M. Nord, K. Basu, F. Pacaud, P. A. R. Ade, A. N. Bender, B. A. Benson et al., Multi-frequency imaging of the galaxy cluster Abell 2163 using the Sunyaev-Zel'dovich effect, Astronomy and Astrophysics 506 (Nov., 2009) 623-636, [0 902.2131$].$

[11] H. Bourdin, M. Arnaud, P. Mazzotta, G. W. Pratt, J.-L. Sauvageot, R. Martino et al., A2163: Merger events in the hottest Abell galaxy cluster. II. Subcluster accretion with galaxy-gas separation, Astronomy and Astrophysics 527 (Mar., 2011) A21, [1011.3154].

[12] G. Soucail, Dark matter distribution in the merging cluster Abell 2163, Astronomy and Astrophysics 540 (Apr., 2012) A61, [1203.0229].

[13] M. S. Owers, P. E. J. Nulsen, W. J. Couch and M. Markevitch, A High Fidelity Sample of Cold Front Clusters from the Chandra Archive, Astrophysical Journal 704 (Oct., 2009) 1349-1370, [0 909.2645$]$.

[14] T. Herbig and M. Birkinshaw, Discovery of a Radio Halo Source in Abell 2163, in American Astronomical Society Meeting Abstracts, vol. 26 of Bulletin of the American Astronomical Society, p. 1403, Dec., 1994.

[15] L. Feretti, E. Orrù, G. Brunetti, G. Giovannini, N. Kassim and G. Setti, Spectral index maps of the radio halos in Abell 665 and Abell 2163, Astronomy and Astrophysics 423 (Aug., 2004) 111-119, [astro-ph/ 0404283 ].

[16] L. D. Landau and E. M. Lifshitz, Fluid mechanics. 1959.

[17] H. Kang and D. Ryu, Curved Radio Spectra of Weak Cluster Shocks, Astrophysical Journal 809 (Aug., 2015) 186, [1505.04256].

[18] H. Kang and D. Ryu, Re-acceleration Model for Radio Relics with Spectral Curvature, Astrophysical Journal 823 (May, 2016) 13, [1602.03278].

[19] R. J. van Weeren, H. J. A. Röttgering, H. T. Intema, L. Rudnick, M. Brüggen, M. Hoeft et al., The "toothbrush-relic": evidence for a coherent linear 2-Mpc scale shock wave in a massive merging galaxy cluster?, Astronomy and Astrophysics 546 (Oct., 2012) A124, [1209.2196]. 
[20] S. Gabici and P. Blasi, Nonthermal Radiation from Clusters of Galaxies: The Role of Merger Shocks in Particle Acceleration, Astrophysical Journal 583 (Feb., 2003) 695-705, [astro-ph/0207523].

[21] M. Markevitch, A. H. Gonzalez, L. David, A. Vikhlinin, S. Murray, W. Forman et al., A Textbook Example of a Bow Shock in the Merging Galaxy Cluster 1E 0657-56, Astrophysical Journal Letters 567 (Mar., 2002) L27-L31, [astro-ph / 0110468 ].

[22] M. Markevitch, F. Govoni, G. Brunetti and D. Jerius, Bow Shock and Radio Halo in the Merging Cluster A520, Astrophysical Journal 627 (July, 2005) 733-738, [astro-ph/0412451].

[23] G. Macario, M. Markevitch, S. Giacintucci, G. Brunetti, T. Venturi and S. S. Murray, A Shock Front in the Merging Galaxy Cluster A754: X-ray and Radio Observations, Astrophysical Journal 728 (Feb., 2011) 82, [1010 . 5209].

[24] H. Akamatsu and H. Kawahara, Systematic X-Ray Analysis of Radio Relic Clusters with Suzaku, Publications of the Astronomical Society of Japan 65 (Feb., 2013) , [1112 . 3030].

[25] L. Feretti, G. Giovannini, F. Govoni and M. Murgia, Clusters of galaxies: observational properties of the diffuse radio emission, Astronomy and Astrophysics Reviews 20 (May, 2012) 54, [1205 . 1919].

[26] G. Brunetti and T. W. Jones, Cosmic Rays in Galaxy Clusters and Their Nonthermal Emission, International Journal of Modern Physics D 23 (Mar., 2014) 1430007-98, [1401.7519].

[27] M. Murgia, P. Parma, K.-H. Mack, H. R. de Ruiter, R. Fanti, F. Govoni et al., Dying radio galaxies in clusters, Astronomy and Astrophysics 526 (Feb., 2011) A148, [1011.0567].

[28] R. Blandford and D. Eichler, Particle acceleration at astrophysical shocks: A theory of cosmic ray origin, Physical Report 154 (Oct., 1987) 1-75.

[29] G. A. Ogrean, M. Brüggen, R. J. van Weeren, H. Röttgering, J. H. Croston and M. Hoeft, Challenges to our understanding of radio relics: X-ray observations of the Toothbrush cluster, Monthly Notices of the Royal Astronomical Society 433 (July, 2013) 812-824, [1303.1533].

[30] M. Trasatti, H. Akamatsu, L. Lovisari, U. Klein, A. Bonafede, M. Brüggen et al., The radio relic in Abell 2256: overall spectrum and implications for electron acceleration, Astronomy and Astrophysics 575 (Mar., 2015) A45, [1411.1113].

[31] A. Stroe, T. Shimwell, C. Rumsey, R. van Weeren, M. Kierdorf, J. Donnert et al., The widest frequency radio relic spectra: observations from $150 \mathrm{MHz}$ to $30 \mathrm{GHz}$, Monthly Notices of the Royal Astronomical Society 455 (Jan., 2016) 2402-2416, [1510. 06739 ].

[32] T. A. Enßlin and Gopal-Krishna, Reviving fossil radio plasma in clusters of galaxies by adiabatic compression in environmental shock waves, Astronomy and Astrophysics 366 (Jan., 2001) 26-34, [astro-ph / 0011123$].$ 
[33] T. A. Ensslin, P. L. Biermann, U. Klein and S. Kohle, Cluster radio relics as a tracer of shock waves of the large-scale structure formation, Astronomy and Astrophysics 332 (Apr., 1998) 395-409, [astro-ph/9712293].

[34] F. de Gasperin, R. J. van Weeren, M. Brüggen, F. Vazza, A. Bonafede and H. T. Intema, A new double radio relic in PSZ1 G096.89+24.17 and a radio relic mass-luminosity relation, Monthly Notices of the Royal Astronomical Society 444 (Nov., 2014) 3130-3138, [1408.2677].

[35] S. Giacintucci, T. Venturi, G. Macario, D. Dallacasa, G. Brunetti, M. Markevitch et al., Shock acceleration as origin of the radio relic in A 521?, Astronomy and Astrophysics $\mathbf{4 8 6}$ (Aug., 2008) 347-358, [0803.4127].

[36] H. Bourdin, P. Mazzotta, M. Markevitch, S. Giacintucci and G. Brunetti, Shock Heating of the Merging Galaxy Cluster A521, Astrophysical Journal 764 (Feb., 2013) 82, [1302.0696].

[37] N. E. Kassim, T. E. Clarke, T. A. Enßlin, A. S. Cohen and D. M. Neumann, Low-Frequency VLA Observations of Abell 754: Evidence for a Cluster Radio Halo and Possible Radio Relics, Astrophysical Journal 559 (Oct., 2001) 785-790.

[38] A. Finoguenov, C. L. Sarazin, K. Nakazawa, D. R. Wik and T. E. Clarke, XMM-Newton Observation of the Northwest Radio Relic Region in A3667, Astrophysical Journal 715 (June, 2010) 1143-1151, [1004.2331].

[39] S. Dasadia, M. Sun, C. Sarazin, A. Morandi, M. Markevitch, D. Wik et al., A Strong Merger Shock in Abell 665, Astrophysical Journal Letters 820 (Mar., 2016) L20, [1603. 01271$].$

[40] M. S. Owers, P. E. J. Nulsen, W. J. Couch, C.-J. Ma, L. P. David, W. R. Forman et al., A Merger Shock in A2034, Astrophysical Journal 780 (Jan., 2014) 163, [1311.5561].

[41] T. W. Shimwell, M. Markevitch, S. Brown, L. Feretti, B. M. Gaensler, M. Johnston-Hollitt et al., Another shock for the Bullet cluster, and the source of seed electrons for radio relics, Monthly Notices of the Royal Astronomical Society 449 (May, 2015) 1486-1494, [1502.01064]. 